Revue d'histoire de l'Amérique française

REVUE D.HISTOIRE DE L'AMÉRIQUE FRANÇAISE

\title{
Une intervention à Rome dans la lutte pour le suffrage féminin au Québec (1922)
}

\section{Luigi Trifiro}

Volume 32, numéro 1, juin 1978

URI : https://id.erudit.org/iderudit/303670ar

DOI : https://doi.org/10.7202/303670ar

Aller au sommaire du numéro

Éditeur(s)

Institut d'histoire de l'Amérique française

ISSN

0035-2357 (imprimé)

1492-1383 (numérique)

Découvrir la revue

Citer cet article

Trifiro, L. (1978). Une intervention à Rome dans la lutte pour le suffrage

féminin au Québec (1922). Revue d'histoire de l'Amérique française, 32(1), 3-18.

https://doi.org/10.7202/303670ar d'utilisation que vous pouvez consulter en ligne.

https://apropos.erudit.org/fr/usagers/politique-dutilisation/ 


\section{UNE INTERVENTION À ROME DANS LA LUTTE POUR LE SUFFRAGE FÉMININ AU QUÉBEC (1922)}

LUIGI TRIFIRO

Université de Sherbrooke

\section{La situation de la lutte pour le suffrage féminin au Québec en 1922}

$\mathrm{Au}$ lendemain de la première Guerre mondiale, le Canada, à l'instar de la France, de l'Angleterre et des États-Unis, pour ne citer que les pays immédiatement associés au développement du Canada, se voit confronter à des réalités qui obligent le gouvernement canadien et les gouvernements provinciaux à s'adapter à un nouveau mode de vie. Au point de vue économique, l'industrialisation attire vers l'usine une main-d'œuvre féminine. Jusque-là, la famille avait été, pour la femme, le lieu privilégié et protecteur d'où il lui était interdit de sortir. La société en mutation amène une nouvelle conception du rôle de la femme. Et c'est justement cette conception qui va ouvrir les portes du bureau de scrutin à la Canadienne.

Cependant, ce vote, la femme ne l'acquerra que graduellement. Les femmes de l'Ouest canadien seront les premières à obtenir le droit de vote. En effet, le Manitoba voit les efforts de Lillian Thomas, de Mary Crawford et de Nellie McClung récompensés en janvier 1916. Le mouvement est déclenché. D'autres provinces vont suivre: la Saskatchewan et l'Alberta en mars et avril de la même année. L'Ontario et la Colombie-Britannique leur emboîtent le pas en avril 1917, suivis de la Nouvelle-Écosse en avril 1918. Toutes ces initiatives provinciales forcent le gouvernement fédéral à accorder le suffrage féminin aux Canadiennes en mai $1918^{1}$. L'année suivante,

1 Le gouvernement canadien avait déjà accordé le droit de vote à un groupe de femmes durant la guerre: les infirmières dans l'armée, les femmes qui avaient de proches parents sous les drapeaux. Voir Catherine Lyle CLEVERDON, The Women Suffrage Movement in Canada (U.T.P. Toronto, 1950), 324 p. 
en avril 1919, le Nouveau-Brunswick accorde à son tour la «libération politique» aux femmes de la province, imité en cela par l'île-du-Prince-Édouard trois ans plus tard, en mai 1922.

En 1922, seul le Québec retarde à passer la législation qui ferait des femmes québécoises des «citoyennes» à part entière au même titre que toutes les autres femmes du Canada.

Mais le droit de vote aux femmes est un sujet tabou au Québec dans les années 20. Revendiquer le suffrage féminin, c'est encourir le risque de la réprobation de certaines autorités, notamment l'Épiscopat. En effet, celui-ci ne peut admettre la discussion sur le sujet. Se rappelant cette époque, le Père Ceslas Forest écrit: "Il existait à ce moment-là une quantité de choses qu'il n'était pas même permis de discuter: l'enseignement secondaire, le suffrage féminin, l'enseignement obligatoire, l'évolution, etc, etc. ${ }^{2}$ » Cependant, quelques femmes osent, malgré l'interdit, aborder le sujet du suffrage féminin au Québec, et certains journaux en font mention, sans prendre position cependant. Mais l'on sent bien dans la province que le féminisme divise déjà les esprits. En effet, la position ferme de l'Église et de l'État sur la question découle logiquement d'une mentalité conservatrice qui croit au rôle strictement familial de la femme, non sans une certaine hypocrisie. Dans une lettre à Marie LacosteGérin-Lajoie, Athanase David écrivait à ce propos

(...) l'argument (de la déchéance de la femme qui s'occupe de politique) est-il bien sérieux, quand on songe que depuis près de cent ans la société a vu sans s'émouvoir la femme prendre le chemin de la manufacture, dans quel qu'état qu'elle se trouvât, et que les hommes ont bénéficié du travail industriel de la femme pour ériger des fortunes que seuls, ils contrôlaient par les lois qu'ils passaient. ${ }^{3}$

C'est dans cet esprit qu'un affrontement se prépare entre les tenants des deux positions sur le suffrage féminin. À l'automne 1921, un groupe de femmes fondent le Comité provincial pour le Suffrage féminin qui doit voir à faire déposer un projet de loi à l'Assemblée Législative du Québec le plus tôt possible. En opposition aux féministes qui luttent pour l'égalité politique de la femme québécoise,

2 Journal personnel du Père Ceslas Forest, o.p. Ce manuscrit inédit nous fut gracieusement fourni par le Père Benoît Lacroix, o.p. Le Père Forest est l'un de ceux qui ont pris une part active dans la lutte du suffrage féminin au Québec.

3 A. David, Lettre à Marie Lacoste-Gérin-Lajoie, 11 février 1922. Archives des Sœurs Notre Dame du Bon Conseil. (Désormais, Archives S.N.D.B.C.) 
un Comité de Propagande contre le Suffrage féminin est mis sur pied, encouragé par Mgr Paul-Eugène Roy, évêque coadjuteur de Québec qui tient à manifester publiquement son opposition au vote des femmes. $\grave{A}$ cet effet, il rédige une lettre pastorale dont le titre est sans équivoque: «Lettre épiscopale pour encourager la campagne anti-suffragiste dans Québec. Un attentat contre les traditions fondamentales de notre race et de notre foi ». ${ }^{4}$ Tous les évêques de la province appuient cette lettre.

Le premier ministre Taschereau suit la même voie quand il se déclare nettement contre le suffrage féminin:

Dans aucun pays français ou latin (...) on n'a accordé le droit de suffrage aux femmes. Je me demande ce que pourra gagner la femme en ayant le droit de vote. Sa place n'est pas dans la politique, c'est au foyer, dans la famille où elle peut exercer la meilleure influence. En se mêlant aux luttes politiques, la femme perdra peut-être le respect qu'on lui doit. ${ }^{5}$

Mais s'enfermer dans une attitude aussi rigide, c'est provoquer inévitablement des conflits. Voilà précisément ce qui arrive au Québec durant l'année 1922 quand les tenants de l'égalité politique s'expriment en paroles et en gestes ${ }^{6}$.

Déjà appelés à participer à la vie politique du Canada, les membres du Comité provincial pour le Suffrage féminin lancent en 1922, un vibrant appel à la logique: si les femmes votent au fédéral pourquoi ne voteraient-elles pas au provincial? Mais le Comité entretient pour un temps de vains espoirs puisque l'Église du Québec veille sur la tradition. Le nouveau «féminisme réformiste catholique », selon l'expression de Michèle Jean ${ }^{7}$, n'est pas très sûr pour

4 Mgr P.-E. Roy, «Lettre épiscopale pour encourager la campagne antisuffragiste dans Québec», in La Presse, 18 février 1922, 1. Le Père Forest rapporte à ce sujet dans son manuscrit que Marie Lacoste-Gérin-Lajoie «étant allée cette année-là au Congrès international des associations féminines catholiques à Rome, y apprit que Mgr Roy avait été réprimandé pour avoir fait intervenir la religion dans cette question."

5 L.-A. Taschereau, "Les femmes ne doivent pas se mêler de politique», in L'Enseignement Primaire, 43 (mars 1922): 456. Antonin Dupont consacre un chapitre au suffrage féminin dans son volume Les relations entre l'Église et l'État sous Louis-Alexandre Taschereau, 1920-1936 (Montréal, Guérin, 1973), 175-195.

6 Pour une analyse plus détaillée de la question du suffrage féminin en 1921-1922, voir notre mémoire de maîtrise, La crise de 1922 dans la lutte pour le suffrage féminin au Québec (Université de Sherbrooke, 1976), 113 p.

7 Michèle Jean, Québécoises du XXe siècle (Montréal, Ed. du Jour, 1974), 19. 
l'Église du Québec. En effet, celle-ci craint qu'il mine les bases de la société québécoise par ses exigences et ses revendications. L'opposition que lui fait l'Épiscopat empêche les partisans du suffrage féminin d'espérer atteindre leur objectif. C'est pourquoi, après de vaines démarches auprès de l'Épiscopat et de l'État, Marie Lacoste-Gérin-Lajoie, présidente de la Fédération Nationale SaintJean-Baptiste (F.N.S.J.B.) et co-présidente du Comité provincial pour le Suffrage féminin, croit nécessaire de recourir à des instances supérieures. À cette fin, elle ira chez le Délégué apostolique puis à Rome pour tenter de faire lever les barrières qui empêchent toute action.

C'est cet ultime recours qui retient notre attention dans les pages suivantes.

Le 15 mars 1922, Mgr Pietro di Maria, délégué apostolique au Canada, reçoit en audience la présidente de la F.N.S.J.B. Il lui recommande d'aller à Rome afin d'obtenir une directive pontificale au sujet du suffrage féminin, en ayant soin, auparavant, de faire parvenir au Souverain Pontife, par l'intermédiaire de $\mathrm{Mgr}$ Gauthier, un document sur la question. Copie de ce document devra être transmise à la présidente de l'Union internationale des Ligues catholiques féminines (U.I.L.C.F.) ainsi qu'à la présidente de l'Union d'Italie, deux organismes très influents à Rome. Voulant calmer les appréhensions de Marie Lacoste-Gérin-Lajoie, le Délégué apostolique affirme que la Fédération ne relève que de l'évêque de Montréal. Rendant compte de son entrevue à la Fédération, la présidente rapporte: «Il recommande de se tenir en relation constante avec notre évêque, et déclare que nous pouvons user de toute la liberté d'action que celui-ci nous laisse conséquemment, dans les circonstances actuelles. ${ }^{8}$ " Informée par Mgr Gauthier qu'il va lui obtenir une audience privée avec le Pape et présenter le document sur le suffrage préparé à cet effet, Marie Lacoste-Gérin-Lajoie écrit: «Tout cela est providentiel ${ }^{9}$. La Fédération s'en remet donc à la décision pontificale.

Préparant son voyage à Rome, la présidente de la F.N.S.J.B. écrit, dès le 21 mars, à la comtesse Wodzicka, présidente de l'U.I.L.C.F. et de l'Union de Pologne, pour lui demander de la pré-

8 Compte rendu de la visite au Délégué apostolique par Marie LacosteGérin-Lajoie. Brouillon extrait des Minutes de la Fédération, (1922), cartable II, cahier noir intitulé Correspondance F.N.S.J.B., Archives S.N.D.B.C.

$9 \quad$ Ibid. Le souligné est de Marie Lacoste-Gérin-Lajoie elle-même. 
senter aux autorités romaines et d'obtenir un mot du Pape en faveur de l'action politique de la femme ${ }^{10}$. Puis, toujours selon le vœu du Délégué apostolique, elle adresse à la marquise Madeline Patrizi, présidente de l'Union des Femmes catholiques d'Italie et secrétaire de l'U.I.L.C.F., une lettre où elle expose les difficultés que rencontrent dans la province de Québec les partisans du suffrage féminin et, par conséquent, de l'affranchissement politique des femmes. Elle rappelle les faits, insistant sur l'importance des cours d'instruction civique qui, dit-elle, "produisirent un véritable éveil de l'esprit public chez la femme ${ }^{11}$. Elle ne manque pas de rappeler l'intervention publique de Mgr Roy, en remarquant que «sa lettre (...) fit autorité bien en dehors de son diocèse ${ }^{12}$. Elle raconte enfin son entrevue avec Mgr Gauthier et résume ainsi la position de l'archevêque de Montréal:

Bien que Mgr Gauthier ne soit pas personnellement un apôtre du suffrage féminin, cependant avec la science et la droiture qui le caractérisent, il m'éclaira avec bonté sur le sens profond de la question et respecta notre liberté d'action. ${ }^{13}$

Après avoir fait l'état de la question, Marie Lacoste-Gérin-Lajoie demande à la marquise Patrizi de l'aider à être reçue en audience privée par le Pape.

...il nous reste aujourd'hui la perspective de recourir à Rome, et c'est bien là que nous plaçons notre espoir, sachant la direction qui a été donnée par le Saint-Siège depuis quelques années aux femmes d'Italie, d'Espagne et de différents pays, nous espérons en obtenir une semblable. ${ }^{14}$

$\mathrm{Au}$ courant des démarches de Marie Lacoste-Gérin-Lajoie auprès des autorités romaines, le journal L'Action Catholique se demande jusqu'où les femmes vont aller dans leurs revendications. Pour cet organe proche de l'Archevêché de Québec, il est malvenu d'aller chercher auprès d'instances supérieures une directive autre que celle des évêques de la province. Le journaliste dénonce ces femmes qui, devant l'attitude négative des autorités religieuses qué-

10 Marie Lacoste-Gérin-Lajoie, Lettre à la comtesse Wodzicka, 21 mars 1922, archives S.N.D.B.C.

11 Marie Lacoste-Gérin-Lajoie, Lettre à la marquise Patrizi, 16 avril 1922, 4, archives S.N.D.B.C.

12 Ibid., 6.

13 Ibid., 7.

14 Ibid., 8-9. 
bécoises concernant le suffrage féminin, vont aller «ennuyer» le pape avec leur problème. "On voit, écrit-il, quel cas font ces dames, parce qu'elles sont embarquées ou presque dans la galère politique, des enseignements et directions de leurs évêques. Voudront-elles maintenant faire une église dans l'Église? ${ }^{15}$ Leur attitude frôle l'hérésie, voire le schisme.

Encouragée par le Délégué apostolique, la présidente de la F.N.S.J.B. adresse, au nom de son organisme, une lettre au Pape dans laquelle elle lui explique la situation du mouvement en faveur du suffrage féminin du Québec. Après avoir tracé un bref historique de la fondation de la Fédération, elle en fait connaitre les objectifs socio-politiques puis décrit les difficultés auxquelles se heurtent les membres de la Fédération. Elle exprime enfin le désir de voir le Souverain Pontife éclairer leur conscience et les guider dans la voie où elles se sont engagées. Elle écrit:

Sans une direction pontificale qui donne confiance à notice popuilation, il ne semble pas possible de faire effectivement l'éducation civique de la femme dans la Province de Québec. ${ }^{16}$

Puis, rappelant que les protestantes participent activement à la vie politique, elle joue la carte de l'influence catholique:

Ce fait est extrêmement grave puisque Québec est le foyer du catholicisme au Canada. L'abstention des femmes catholiques de la vie publique à l'heure où les protestantes siègent au Parlement et influencent la législation ne serait-elle pas une omission coupable ? ${ }^{17}$

Conscient de la situation dans laquelle il se trouve et ne voulant brusquer personne, Mgr Pizzardo, secrétaire de Pie XI, demande à Marie Lacoste-Gérin-Lajoie ce qu'elle espère des autorités romaines dans les difficultés que traverse la F.N.S.J.B. au sujet du suffrage féminin $^{18}$. La présidente de la Fédération lui répond le 24 mai 1922 que les cours d'éducation civique sous le patronage de l'Université

15 Anonyme, "Leur ambition", in L'Action Catholique, 26 avril 1922, coupure de journal trouvée dans les papiers de Marie Lacoste-Gérin-Lajoie. Archives S.N.D.B.C.

16 Marie Lacoste-Gérin-Lajoie, Lettre à Sa Sainteté Pie XI, $1^{\text {er }}$ mai 1922, 5-6, dans Papiers très personnels. Archives S.N.D.B.C.

17 Ibid., 6.

18 Marie Lacoste-Gérin-Lajoie, Lettre à Mgr Pizzardo, 24 mai 1922. Cette lettre porte la mention «lettre Rome - très personnelle, très important à conserver.» Archives S.N.D.B.C. 
de Montréal vont se développer «selon la mesure de l'encouragement que la Fédération recevra du Souverain Pontife à ce sujet. Mgr Gauthier (...) attend le résultat des événements de Rome, pour prendre une décision à ce sujet ${ }^{19} \mathrm{Ce}$ que Marie Lacoste-GérinLajoie ne peut dire, sans doute, c'est que Mgr Gauthier ne saurait aller à l'encontre des directives des autres membres de l'épiscopat sans l'appui de Rome.

\section{Marie Lacoste-Gérin-Lajoie et le Congrès de l'U.I.L.C.F. à Rome, mai 1922}

Précédées par ces lettres aux autorités romaines et aux responsables du Congrès, c'est dans un esprit de confiance absolue que la présidente de la Fédération et la secrétaire, Georgette LeMoyne, quittent le Canada, au début du mois de mai, pour le congrès de l'U.I.L.C.F. Pour elles, le Congrès est l'occasion rêvée de gagner à leur cause l'épiscopat québécois en obtenant «la bénédiction» des autorités romaines sur leur mouvement et sur la campagne entreprise en faveur du suffrage féminin au Québec. Marie Lacoste-GérinLajoie le dit très clairement à son mari, dans une lettre du 14 mai 1922:

(...) je ne laisserai pas Rome avant d'épuiser nos moyens de succès et je suis même décidée de sacrifier le reste du voyage plutôt que d'échouer dans les démarches que j'ai entreprises.

(...) Seul l'objet qui me guide et m'entraîne vers Rome remplit donc ma vie en ce moment. Puisse le ciel venir à mon aide à cette heure si importante de ma vie. ${ }^{20}$

Quinze pays sont représentés au Congrès et une centaine de déléguées participent aux délibérations. Le Pape y délègue le Cardinal Merry del Val. Quatre commissions d'étude y sont créées, la quatrième se penchant sur la formation de la femme à ses devoirs civiques $^{21}$. Marie Lacoste-Gérin-Lajoie participe aux travaux de cette commission, selon les objectifs précis de son pèlerinage à Rome. Au terme de ses travaux, la quatrième commission émet trois vœux

\footnotetext{
19 Ibid.

20 Marie Lacoste-Gérin-Lajoie, Lettre à Henri Gérin-Lajoie, 14 mai 1922. Archives S.N.D.B.C.

21 La première commission d'étude portait sur la propagation de la foi; la seconde étudiait les moyens à prendre pour lutter contre l'immoralité des modes, du cinéma et du théâtre; la troisième commission examinait le problème de la traite des blanches.
} 
précédés de cinq considérants, qu'il vaut la peine de reproduire intégralement:

I. Considérant que le droit et l'exercice du suffrage électoral de la femme ne sont pas exclus par le droit divin ou par une loi positive générale du droit ecclésiastique, comme, au contraire, c'est le cas en ce qui regarde les ordres sacrés et le gouvernement de l'Église;

II. Considérant que la vie morale d'un pays dépend de sa législation et que par conséquent la responsabilité directe de chaque électeur est engagée dans le choix des législateurs;

III. Considérant que ces responsabilités impliquent celles d'une préparation religieuse, morale et civique ;

IV. Considérant que le suffrage féminin est déjà en vigueur dans plusieurs pays et pourra l'être demain bon gré mal gré, partout et que, quelle que soit la forme du suffrage, l'opinion publique étant en force, la femme ne doit pas s'en désintéresser;

V. Considérant que la femme peut apporter une compétence spéciale en ce qui concerne les lois familiales, scolaires et d'hygiène,

Le Conseil émet les vœux suivants:

I. Que les femmes catholiques de tous les pays comprennent leur responsabilité morale en face du suffrage électoral, quel qu'en soit le mode;

II. Que les femmes se préparent à leur rôle par une formation morale et religieuse et civique qui les rende aptes, le cas échéant, à cet apostolat ;

III. Que toute nouvelle initiative, sur le terrain du suffrage féminin, soit soumise d'avance dans chaque pays à l'approbation de l'Épiscopat ${ }^{22}$.

Ce texte règle définitivement la question de doctrine si souvent soulevée au Québec depuis le début de la lutte pour le suffrage féminin. Il semble ne rester que la question d'opportunité, dont l'épiscopat demeure seul juge d'après le troisième vœu du Conseil. Pour sa part, le deuxième vœu, touchant à la préparation civique de la femme,

22 Rapport de l'Union Internationale des Ligues catholiques féminines, 5e Conseil International, s.l. n.d., conservé aux Archives de la F.N.S.J.B. Voir aussi «Le suffrage féminin, les congressistes de Rome et l'épiscopat, in Le Devoir, 29 juin 1922, 1. 
devient un appui précieux aux cours d'instruction civique mis sur pied par la Fédération.

Mais les vœux de la commission laissent Marie Lacoste-GérinLajoie fort déçue. Elle espérait plus qu'un énoncé de principes. Elle attendait une prise de position ferme qui ne laisserait place à aucun malentendu. Elle comprend cependant l'attitude des déléguées qui ne vivent pas les mêmes problèmes que les Québécoises en regard des droits politiques de la femme. Aussi écrit-elle dans La Bonne Parole, organe de la Fédération:

(...) le suffrage ne fut point discuté à son mérite au congrès, probablement parce que ce milieu était largement composé de déléguées venant de pays où les femmes exercent leurs droits politiques ou les ont inscrits au programme des partis politiques... ${ }^{23}$

Elle s'explique le caractère imprécis des résolutions:

Il faut avouer toutefois que les résolutions de la IV e commission n'ont pas cette netteté et cette précision qu'on remarque dans les autres résolutions du congrès. On sent que l'on est sur un terrain où les ménagements sont encore nécessaires et où l'accord entre les catholiques n'est pas encore consommé. ${ }^{24}$

Malgré tout, Marie Lacoste-Gérin-Lajoie apprécie les contacts qu'elle a pu établir à Rome, durant le Congrès. À ce point de vue, ses objectifs sont atteints. Elle l'écrit à son mari le 2 juillet:

Je suis très satisfaite de ce que $\mathrm{j}$ 'ai obtenu et de l'influence que j'ai acquise au Vatican. Je t'ai dit déjà qu'on me demande de rester en correspondance avec le secrétaire. Mes vues auront donc la chance d'être comprises, et $j$ 'espère beaucoup de l'avenir. ${ }^{25}$

De retour au Canada, la présidente de la F.N.S.J.B. rend compte à Mgr Gauthier de son voyage à Rome ${ }^{26}$. Elle se montre tout heureuse de ce que les résolutions du congrès sur la nécessité

23 Marie Lacoste-Gérin-Lajoie, "Rapport du Congrès de l'Union Internationale des Ligues catholiques féminines ", in La Bonne Parole (sept. 1922): 3.

24 Ibid.: 4.

25 Marie Lacoste-Gérin-Lajoie, Lettre à Henri Gérin-Lajoie, 2 juillet 1922. Archives S.N.D.B.C.

26 Marie Lacoste-Gérin-Lajoie, Lettre à Mgr Pizzardo, 10 août 1922, 1. La date exacte de la rencontre nous est inconnue. Les minutes de la Fédération rapportent l'événement sans en préciser la date. 
de bien préparer la femme à son nouveau rôle permettent à la Fédération de continuer à dispenser les cours d'instruction civique. En même temps, ces résolutions engagent en quelque sorte l'évêque de Montréal à répandre davantage de tels cours. À ce propos, elle écrit en ces termes à Mgr Pizzardo:

Tout ce qui s'est passé confirme la justesse de son [Mgr Gauthier] attitude dans cette question; il est décidé de créer en notre pays une mentalité chrétienne à ce sujet et de transposer l'apostolat social dans ce champ d'action qui s'ouvre au dévouement de la femme, celle-ci jouissant déjà de ses droits politiques au fédéral. Je le reverrai pour entrer dans le détail du programme et favoriser l'expansion de l'instruction civique de la femme. [...] Sa Grandeur partage maintenant nos espérances et s'associe à notre joie. ${ }^{27}$

En suivant les directives de son évêque, la F.N.S.J.B. a donc eu une action qui est conforme aux directives données au monde catholique par l'U.I.L.C.F., sous l'autorité de Rome. La Fédération n'en est que plus heureuse et comprend que l'éducation civique de la femme doit se poursuivre dans le Québec et ce, malgré les embûches de toutes sortes qui peuvent surgir.

\section{L'intervention d'Henri Bourassa au congrès de l'U.I.L.C.F.}

Les cours d'instruction civique vont continuer à se donner, il est vrai, mais le suffrage n'est pas acquis pour autant. C'est pourtant la raison d'être du Comité provincial pour le Suffrage féminin. En conséquence, la F.N.S.J.B. qui a fait sienne cette priorité, se trouve dans un dilemme. Le Congrès de Rome a approuvé la participation des femmes à la vie publique mais une restriction très importante, du moins pour le Québec, a été ajoutée par la $\mathrm{III}^{\mathrm{e}}$ résolution du Conseil: "Que toute nouvelle initiative, sur le terrain du suffrage féminin, soit soumise d'avance dans chaque pays à l'approbation de l'Épiscopat.» Déjà à Rome même, pendant le Congrès, Marie Lacoste-Gérin-Lajoie, à l'instar de quelques autres déléguées, s'est montrée étonnée que cette $\mathrm{III}^{\mathrm{e}}$ résolution n'ait pas été "discutée» et qu'elle ait été «introduite au dernier moment, en séance solennelle ${ }^{28}$. Marie Lacoste-Gérin-Lajoie, qui a participé aux travaux de

27 Ibid.: 2.

28 Marie Lacoste-Gérin-Lajoie, Lettre à la comtesse Wodzicka, 6 juillet 1922, 2. Archives S.N.D.B.C. 
la IV Commission sur les devoirs civiques de la femme, ne peut comprendre cette addition surprise aux conclusions de la Commission. Elle dit alors son étonnement à la marquise Patrizi, présidente de la IV ${ }^{e}$ Commission qui lui explique que c'est Son Éminence le Cardinal Merry del Val qui a ajouté cette troisième résolution.

Je ne m'attendais à aucun changement entre la séance close et la séance ouverte. Mais voilà qu'au dernier moment, lorsque nous étions déjà dans la Salle du Congrès, Son Éminence me donna, écrite de sa main, la $3^{\text {ème }}$ conclusion concernant le devoir de s'en remettre aux évêques pour tout mouvement se rapportant au suffrage.

Et d'ailleurs, savons-nous si le Cardinal Légat parlait de son propre chef ou s'il nous transmettait un désir du Saint-Père ? ${ }^{29}$

Ouvrons ici une parenthèse pour expliquer que toutes les résolutions votées par les déléguées au Congrès de Rome sont antérieurement étudiées, acceptées et rédigées en «séance close», qui sont "réservées seulement aux déléguées des ligues affiliées» ${ }^{30}$. Ces résolutions doivent obligatoirement être présentées en «séance solennelle» et votées par toutes les délégations. C'est en «séance close» que se préparent et se décident les grandes orientations des Ligues catholiques féminines, alors que la «séance solennelle» doit ou bien entériner ou bien amender, très exceptionnellement cependant, les décisions prises par les ligues affiliées en «séance close».

Marie Lacoste-Gérin-Lajoie est très insatisfaite des conclusions du Congrès. Elle quitte Rome, résolue à pousser plus loin son enquête sur un événement qui menace de paralyser entièrement son action en faveur du suffrage féminin. Comme présidente de deux organismes importants voués à la promotion et aux intérêts de la femme, elle considère le vote féminin comme un préalable nécessaire à toute action sociale efficace. On le sait et son action le confirme. Pour elle, les conséquences de la $\mathrm{III}^{\mathrm{e}}$ résolution sont donc très graves. Aussi, de retour au Canada, adresse-t-elle une lettre à l'ex-présidente de l'Union internationale, la comtesse Wodzicka, dénonçant le fait que cette $\mathrm{III}^{\mathrm{e}}$ résolution ait été votée "sans que nous ayons eu le temps, écrit-elle, de l'approfondir et de participer

29 Madeleine Patrizi, Lettre à Marie Lacoste-Gérin-Lajoie, 2 août 1922, 1-2. Archives S.N.D.B.C.

30 Rapport de l'Union internationale des Ligues catholiques féminines 5 e Conseil international tenu à Rome du 18 au 23 mai 1922, 6, s.l. n.d. 
à sa rédaction ${ }^{31}$. Elle affirme, concernant les résolutions : «... on m'avait durant le Congrès tenue au courant des modifications qui y seraient apportées sauf la dernière. Voilà ce qui justifie n'est-ce pas des explications...» ${ }^{32}$ Marie Lacoste-Gérin-Lajoie perçoit quelque chose de louche dans toute cette affaire qu'elle tente de tirer au clair.

Que s'est-il donc passé entre la séance close et la séance ouverte du Congrès? Pourquoi le Cardinal Merry del Val a-t-il agi de la sorte, se moquant ainsi de la procédure officielle? Pour la présidente de la Fédération, il y a de quoi être intriguée. Se rappelant les explications déjà fournies à Rome par la comtesse Wodzicka, Marie Lacoste-Gérin-Lajoie lui écrit:

Il faudrait d'ailleurs savoir d'où vient l'inspiration de cette résolution. Quand vous songez aux démarches que «Le Devoir», quotidien de Montréal a faites auprès de vous pour paralyser l'action de l'Union Internationale des Ligues Catholiques Féminines dans cette question du suffrage féminin, cela donne à réfléchir. ${ }^{33}$

On sent chez la présidente la crainte d'une intervention personnelle d'Henri Bourassa qui arpente les corridors du Vatican pendant que se tient le Congrès et dont les relations avec Mgr Merry del Val existent de longue date, depuis le séjour du prélat au Canada à l'occasion de la crise des Écoles du Manitoba en 1897. Et quand on connaît l'attitude de Bourassa sur le suffrage féminin, les soupçons de Marie Lacoste-Gérin-Lajoie nous semblent justifiés ${ }^{34}$.

Depuis mars 1922, le directeur du Devoir séjourne en Europe où il assiste au Congrès Eucharistique International. Vers la fin de son séjour et avant de revenir au Canada, s'ouvre à Rome le cinquième Congrès de l'U.I.L.C.F. Il en profite pour s'y glisser grâce à un «introducteur obligeant», un dénommé Parlowski de l'ambassade de Pologne. C'est alors qu'il rencontre la comtesse Wodzicka, présidente de l'Union Internationale. Celle-ci lui aurait fait certains aveux sur sa conception du suffrage féminin: «Pour ma part, je ne vois pas dans le suffrage un droit de la femme, ni même un avan-

31 Marie Lacoste-Gérin-Lajoie, Lettre à la Comtesse Wodzicka, 6 juillet 1922, 2. Archives S.N.D.B.C.

32 Ibid.

33 Ibid., 2-4.

34 Cf. Susan Mann Trofimenkoff, «Henri Bourassa et la question des femmes", in M. Lavigne et Y. Pinard, Les femmes dans la société québécoise (Montréal, Boréal Express, 1977), 109-124. 
tage ${ }^{35}$ ». Elle ajoute cependant que c'est la guerre qui a imposé le vote à de nombreux pays. Elle explique alors le rôle qui incombe à la femme catholique:

Si nous, femmes catholiques, n'aidons pas les femmes de nos pays respectifs à s'adapter aux conditions qui leur sont faites, tout en respectant les lois et les traditions de l'Église, elles seront la proie des organisations socialistes, neutres ou antireligieuses. Si nous n'entraînons pas les femmes catholiques à user pour le bien général de l'arme du suffrage qu'on leur a mise entre les mains, elles seront amenées à s'en servir pour le mal, ou tout au moins à laisser le champ libre aux agents du mal, hommes et femmes. ${ }^{36}$

Après sa conversation avec la comtesse Wodzicka, Bourassa arrive à la conclusion qu'au Canada et en particulier, au Québec où l'on ne vit pas la situation dangereuse engendrée par la guerre, le suffrage féminin ne peut se justifier: "...un régime imposé par des circonstances particulières de lieu et de temps ne doit pas s'appliquer aux pays où ces circonstances ne se présentent pas ${ }^{37}$. Bourassa essaie donc d'empêcher que la question du suffrage féminin figure au programme du Congrès de Rome ${ }^{38}$. N'obtenant aucun succès de ce côté, il se tourne alors vers le Cardinal Merry del Val et on peut logiquement supposer qu'il lui glisse l'idée de la III ${ }^{\text {ème }}$ résolution qui doit faire échec au mouvement en faveur du vote féminin au Québec. Mais nous pouvons aller plus loin dans notre hypothèse. Le directeur du Devoir aurait-il reçu mandat de certaines autorités religieuses du Québec? On connaît la grande influence de cet homme sur tous les aspects de la vie sociale, politique, religieuse du Canada français. Bourassa a joué et joue encore dans les années 20 , un rôle incontestable. Laïc très engagé, il véhicule les idées ultramontaines de la fin du XIX $\mathrm{X}^{\mathrm{e}}$ siècle et croit à l'autorité de l'Église sur l'État ${ }^{39}$. A Rome, il peut donc être le porte-parole de l'épiscopat québécois.

35 H. Bourassa, Femmes-Hommes ou Hommes et Femmes, Etudes à bâtons rompus sur le féminisme (Montréal, Imprimerie du Devoir, 1925) 83 p. Voir p. 7.

36 Ibid., 7-8.

37 Ibid., 8.

38 Commentaires de Marie Lacoste-Gérin-Lajoie, cartable II, cahier noir intitulé «Correspondance», Archives S.N.D.B.C.

39 Cf. René Durocher, "Un journaliste catholique au $\mathrm{XX}^{\mathrm{e}}$ siècle: Henri Bourassa", in Le laïc dans l'Église canadienne-française de 1830 à nos jours (Montréal, Fides 1972), 185-213. Aussi Jean Drolet, «Henri Bourassa: une analyse de sa pensée", in Idéologies au Canada-français 1900-1929 (Québec, P.U.L. 1974), 223-250. 


\section{Ultimes tentatives de Marie Lacoste-Gérin-Lajoie}

Au lendemain du Congrès de Rome, l'action de la F.N.S.J.B. de Montréal sur la question du suffrage se trouve donc paralysée par la célèbre $\mathrm{III}^{\mathrm{e}}$ résolution. Mais Marie Lacoste-Gérin-Lajoie n'est pas femme à démissionner s'il y a quelque lueur d'espoir. Elle cherche donc une issue qui lui permette de continuer l'action commencée. Très audacieusement, elle suggère donc au Conseil de l'U.I.L.C.F. qui doit bientôt se réunir, de modifier le texte avant sa publication ou même de faire disparaître la résolution purement et simplement $^{40}$. Son argument, c'est que cette résolution n'a pas été discutée antérieurement en séance close. Mais la marquise Patrizi à qui la comtesse Wodzicka a référé la demande refuse toute modification dans le texte: «Demander à son Éminence la suppression de cet article dans les Actes du Congrès ne me semble pas possible. Du moins, ce n'est pas moi qui l'oserais ${ }^{41}$.» Et la nouvelle présidente, F. Steenbergh-Engeringh, qui a aussi reçu la demande de Marie Lacoste-Gérin-Lajoie, écrit très fermement:

Les membres du Bureau (...), constatant que ce vœu ayant été légalement lu, et vôté (sic) avec les autres dans la séance ouverte du Congrès de Rome, ont décidé qu'il serait publié avec les autres, d'autant plus que ce vœu a été ajouté sur la demande formelle du Légat du Saint-Père. ${ }^{42}$

Marie Lacoste-Gérin-Lajoie se trouve devant un problème de taille. D'un côté, elle s'est engagée, au nom de la Fédération, à mettre en vigueur les résolutions passées au Congrès de Rome ${ }^{43}$. De l'autre, elle est entrée dans le mouvement suffragiste au Québec avec l'appui de son évêque, Mgr Gauthier, évêque de Montréal. Les "minutes" de la Fédération sont très explicites à ce sujet: "Madame la Présidente tient à assurer l'assemblée qu'avant chaque démarche (à propos du suffrage), elle a consulté Mgr Gauthier ${ }^{44}$.» En agissant

40 Marie Lacoste-Gérin-Lajoie, Lettre à la comtesse Wodzicka, 6 juillet 1922, Archives S.N.D.B.C.

41 Madeleine Patrizi, Lettre à Marie Lacoste-Gérin-Lajoie, 2 août 1922, 2. Archives S.N.D.B.C.

42 F. Steenbergh-Engeringh, Lettre à Marie Lacoste-Gérin-Lajoie, 10 octobre 1922. Archives S.N.D.B.C.

43 Marie Lacoste-Gérin-Lajoie, Lettre à la comtesse Wodzicka, 6 juillet 1922 , 1. Archives S.N.D.B.C.

44 Minutes du Comité Central d'Étude et d'Action Sociale de la Fédération Nationale Saint-Jean-Baptiste, du 21 mars 1918 au 2 avril 1935, cahier no I, Assemblée du 23 février 1922. 
ainsi, ne pourrait-elle pas répondre aux exigences de la $\mathrm{III}^{\mathrm{e}}$ résolution du Congrès, puisque la Fédération et sa présidente ne relèvent que de l'Evêque de Montréal? Pour elle, «l'épiscopat» pourraitil se limiter à «son» évêque, soit à Mgr Gauthier? Marie LacosteGérin-Lajoie semble prête à accepter ce sens plus «restreint» du terme "épiscopat». Aussi s'avise-t-elle de souligner à la comtesse Wodzicka, ex-présidente de l'U.I.L.C.F., l'ambiguité du texte. Elle lui écrit en ces termes:

Veut-on dire qu'on doit consulter son évêque diocésain, ce qui se fait d'ailleurs en matière grave, ou faut-il attendre pour agir dans les matières se rapportant au suffrage, que l'Épiscopat tout entier se soit rangé à l'idée ? ${ }^{45}$

Puis consciente de la mentalité conservatrice de l'Église en matière sociale, elle ajoute ce commentaire:

(...) à ce compte peu d'institutions catholiques se seraient implantées dans le monde. Vous comprenez qu'il ne faut pas être plus catholique que le pape. On ne relève que de son propre évêque et ce serait ralentir le mouvement du suffrage féminin en certains pays et même le rendre impossible que d'attendre une adhésion générale. Il ne faut pas enlèver aux Ligues Catholiques les initiatives salutaires et civilisatrices, lesquelles sont souvent mal comprises au début et rencontrent beaucoup d'opposition. ${ }^{46}$

On sent que Marie Lacoste-Gérin-Lajoie est prête à tout - sans heurter l'orthodoxie cependant - pour gagner sa cause. Mais une déception l'attend. La comtesse Wodzicka lui répond:

Quant à l'interprétation du passage ajouté par le Card. Merry del Val, je pense personnellement que c'est affaire de l'Évêque diocésain de décider s'il a le droit de conclure au nom de l'Épiscopat tout entier de son pays, il faut évidemment qu'il sache la teneur du passage en question et sa provenance. Du reste, toute initiative pour le suffrage regarde le pays tout entier et pas seulement un diocèse, donc les évêques devraient s'entendre là-dessus. ${ }^{47}$

45 Marie Lacoste-Gérin-Lajoie, Lettre à la comtesse Wodzicka, 6 juillet 1922, 1. Archives S.N.D.B.C.

46 Ibid.

47 Comtesse Wodzicka, Lettre à Marie Lacoste-Gérin-Lajoie, 9 décembre 1922. Archives S.N.D.B.C. 
C'est très clair. Pour que Marie Lacoste-Gérin-Lajoie puisse continuer son action socio-politique en vue du suffrage féminin, il faudrait que tous les évêques québécois «s'entendent là-dessus». La présidente de la F.N.S.J.B. ne peut donc plus agir. En conséquence, en novembre 1922, elle annonce sa démission comme présidente du Comité Provincial pour le Suffrage Féminin.

Avec le départ de Marie Lacoste-Gérin-Lajoie, le Comité tombe dans une période de léthargie. Dans la province, on fait de moins en moins allusion au suffrage féminin qui avait connu pendant l'année 1922 un sommet en retenant l'attention, favorable ou défavorable, de l'État, de l'Église et de l'ensemble des Québécois. Quant à Marie Lacoste-Gérin-Lajoie, qui rentre dans un silence religieux après son échec, elle ne comprend pas qu'au Québec, où la femme a droit de suffrage au niveau fédéral, ne s'applique le vieux principe: "Qui peut le plus peut le moins" ${ }^{48}$.

Pour que le mouvement suffragiste triomphe, il faudra encore vingt ans de luttes aussi acharnées que celle menée par Marie Lacoste-Gérin-Lajoie en 1922. Il faudra que se relâche le lien ÉgliseÉtat, que s'assouplisse la pensée sociale de l'Église québécoise, que se voile la voix grêle de Henri Bourassa, qu'une seconde guerre mondiale renvoie les femmes québécoises à une action civique qui appelle l'émancipation politique. Bref, il faudra peut-être plus qu'une évolution idéologique, il faudra la rencontre d'événements favorables et l'amorce même d'un changement de structures sociales.

48 Marie Lacoste-Gérin-Lajoie, Lettre à la vicomtesse de Vélard, 24 août 1922, 4. Archives S.N.D.B.C. 